\title{
REMOCIÓN DE SEMILLAS, HERBIVORÍA Y RECLUTAMIENTO DE PLÁNTULAS DE BROSIMUM aLICASTRUM (MORACEAE) EN SITIOS CON MANEJO forestal contrastante de la selva Maya, Quintana RoO, México
}

\author{
Gabriel Gutiérrez-Granados ${ }^{1,3}$ y Rodolfo Dirzo ${ }^{2}$ \\ 'Laboratorio de Interacción Planta-Animal, Instituto de Ecología, UNAM. \\ Circuito exterior s/n, Ciudad Universitaria, México D.F. 04510, México. \\ ${ }^{2}$ Department of Biology, Stanford University, 371 Serra Mall, Stanford, CA 94305, USA. \\ ${ }^{3}$ Autor para la correspondencia. Correo-e: ggranados@ecologia.unam.mx
}

\begin{abstract}
Resumen: En este trabajo se evaluó el impacto de la extracción forestal sobre la remoción de semillas, herbivoría y establecimiento de Brosimum alicastrum. Para esto, se utilizaron una serie de exclusiones experimentales colocadas en sitios con manejo y sin manejo forestal. Los resultados muestran que la remoción de semillas y el establecimiento de B. alicastrum fue mayor en las zonas de extracción forestal. La herbivoría fue más intensa en las zonas de reserva y estuvo asociada a una mayor abundancia de $B$. alicastrum lo que sugiere la existencia de efectos denso-dependientes en las zonas de reserva; en las zonas de extracción forestal no existió esta asociación, lo que puede estar explicando el establecimiento diferencial entre condiciones de admanejo. Los resultados indican que la extracción forestal puede alterar procesos denso-dependientes y causar la sobre-representación de algunas especies afectando a largo plazo la diversidad de las selvas.
\end{abstract}

Palabras clave: Extracción maderera; ramón; regeneración; selvas; supervivencia; sotobosque.

\begin{abstract}
The impact of timber extraction was evaluated on Brosimum alicastrum, particularly on seed removal, recruitment and seedling herbivory. A series of experimental exclusions were established in logged and unlogged areas. Results showed that seed removal and recruitment were larger in timber extraction areas. Nonetheless foliar damage was more intense in unlogged areas and it showed a denso-dependency, whereas in timber extraction areas density effects were not significative. Therefore establishment of plants differs according to management conditions. Results suggest that timber extraction affects plant establishment via alteration of denso-dependency what in the long-term would cause an over-representation of some species affecting diversity of tropical rain forests.
\end{abstract}

Key words: Brosimum alicastrum; regeneration; surviving; timber extraction; tropical rainforests; understory.

$\mathbf{L}$ as selvas tropicales presentan una explosión de interacciones bióticas, entre las que sobresalen las interacciones antagónicas entre plantas y animales. De éstas, aquellas establecidas entre plantas y mamíferos terrestres, como son la herbivoría y la depredación de semillas, son reconocidas como clave en el mantenimiento de la alta diversidad arbórea de las selvas (Janzen, 1970; Howe, 1990; DeMattia et al., 2004). Estas interacciones son sensibles a los cambios provocados por las actividades humanas, como la cacería, lo que podría alterar el reclutamiento de plantas y, potencialmente, la estructura y composición florística de las selvas (Wright et al., 2000; Wright y Duber, 2001, Wright, 2003;
Stoner et al., 2007). Sin embargo, los efectos de otras actividades humanas sobre estos procesos ecológicos y que no necesariamente implican la extracción de la fauna, como son la extracción de productos maderables y no maderables de origen vegetal, han sido poco evaluados.

Una actividad económica común en los trópicos es la extracción forestal (Alavalapati y Zarin, 2004). En un sistema de extracción convencional (extracción periódica y selectiva de algunas especies) se daña alrededor del 30 - $70 \%$ de la cobertura forestal (Johns et al., 1996). Esto provoca cambios en la estructura y composición de la vegetación y en el microclima (Webb, 1997; Hall et al., 2003; Makana y To- 
mas, 2005). Esta serie de cambios influye directamente en la comunidad de mamíferos pequeños $(<170 \mathrm{~g}$ ), provocando principalmente un aumento en las densidades de especies de roedores terrestres y en algunos casos una invasión de especies oportunistas de roedores (Malcolm y Ray, 2000; Ochoa, 2000). Por otro lado, se ha documentado que existe en general una disminución en las densidades de mamíferos medianos y grandes, como respuesta a la extracción de su fuente principal de alimento (v. gr., Heydon y Bulloh, 1997) y en general por la cacería que se ve incentivada por el aumento de caminos que facilitan el acceso a zonas remotas de la selva (Peres, 2001). Dado que se ha documentado que la cacería afecta negativamente a los mamíferos medianos y grandes (Wright, 2003; Mendoza y Dirzo, 2007; Stoner $e t$ al., 2007) y que la extracción forestal afecta positivamente a los roedores (Malcolm y Ray, 2000; Ochoa, 2000), se puede esperar que exista un cambio en los procesos en los que intervienen como es la remoción de semillas. Esto es, semillas de tamaño pequeño $(<.1 \mathrm{~cm})$, serán mas consumidas dado la sobre abundancia de roedores pequeños y semillas de tamaño grande $(>1 \mathrm{~cm})$ serán poco consumidas como respuesta a la disminución en la abundancia de mamíferos medianos y grandes (DeMatia et al., 2004; Mendoza y Dirzo, 2007). Dado que se ha propuesto que los roedores pequeños son principalmente depredadores de semillas (Mendoza y Dirzo, 2007), el efecto esperado sería que se afectara de manera negativa el reclutamiento de las plantas con las que interactúan (DeMatia et al., 2004).

Una vez que la semilla ha llegado a un sitio donde pueda germinar y ha escapado de la depredación, ésta se establecerá si las condiciones del sitio son las adecuadas (Muller-Landau et al., 2001). Ya establecidos los juveniles, una de las presiones a las que se enfrentan es la herbivoría (Marquis 1984, Dirzo 1984), la cual se considera actúa bajo efectos denso-dependientes (Schmitz, 2008), por lo que se esperaría que la intensidad de la herbivoría sobre la especie hospedera fuera alta en sitios donde su abundancia es mayor (Janzen, 1970, Carson y Root, 2000; Schmitz, 2008). Las consecuencias de las actividades humanas sobre la herbivoría en general han sido poco evaluadas (Benitez-Malvido et al., 1999). Sin embargo, en zonas de extracción forestal se ha documentado un aumento en la abundancia de herbívoros invertebrados, en respuesta a una mayor disponibilidad de alimento debido a que los claros provocados disparan la regeneración (Fredericksen y Fredericksen, 2002). Con esta información como antecedente es posible predecir que la herbivoría realizada por insectos va a ser en general mayor en las zonas de extracción forestal en comparación con zonas donde no se realice esta actividad. Así, es posible predecir que en aquellos sitios donde la remoción de semillas es alta (extracción forestal), la herbivoria sobre los individuos establecidos va a ser menor que en aquellos sitios donde el establecimiento se da en altas densidades.

La remoción de semillas y la herbivoría son procesos clave en la transición semilla - plántula (Janzen, 1970; Jordano et al., 2007; Schmitz, 2008). Sin embargo, la respuesta de estos procesos a la extracción forestal no ha sido completamente analizada. Debido a esto, en este trabajo evaluamos los efectos de la extracción forestal sobre 1) la remoción de semillas, 2) la herbivoría en función de su desempeño denso-dependiente y 3) el reclutamiento de Brosimum alicastrum.

\section{Materiales y Métodos}

El trabajo fue realizado en el municipio de Carrillo Puerto $\left(88^{\circ} 00^{\prime}-88^{\circ} 20^{\prime} \mathrm{W}\right.$ y $\left.19^{\circ} 00^{\prime}-20^{\circ} 00^{\prime} \mathrm{N}\right)$, Quintana Roo en la región conocida como Zona Maya (Fig. 1). En esta zona el clima es húmedo con una precipitación promedio de 1200 $\mathrm{mm}$, concentrados principalmente en el verano (Junio - Octubre). La vegetación dominante es la selva mediana subperenifolia con parches de selva baja caducifolia (Olmsted y Durán, 1990).

En este sitio existe un programa de extracción forestal el cual ha sido llamado "ecológicamente amigable" (Bray et al., 2003). El sistema de extracción de madera se realiza a través de rodales con cortas programadas por un periodo de 25 años. Es decir, cada uno de los rodales tendrá 25 años de descanso entre corta y corta. El programa considera una serie de tres extracciones por sitio lo que lleva a un manejo total de la selva de 75 años (turno). En algunos ejidos se han seleccionado áreas de reserva, en donde no se ha realizado extracción forestal en al menos los últimos 30 años. Aprovechando esta condición, se seleccionaron para el estudio dos ejidos con ambos tipos de manejo: Señor y Naranjal Poniente. En cada uno de los ejidos se establecieron dos áreas de muestreo, separadas entre si por al menos un kilómetro, por cada condición de manejo; extracción forestal (rodal trabajado en 1996) y reservas. La composición florística y estructura de la selva son similares en ambos sitios y entre tipos de manejo (Gutiérrez-Granados, datos no publicados), por lo que se considera que en general los efectos encontrados se deben a las actividades de manejo y no a otros factores inherentes a los sitios.

Para realizar el presente estudio se decidió utilizar a Brosimum alicastrum Swartz (Moraceae) debido a que es una especie común en las dos condiciones de manejo estudiadas, y en general en la Zona Maya (Olmsted y Durán, 1990) y cuyas semillas son altamente consumidas por mamíferos (Rodríguez-Velázquez et al., 1997). Para cuantificar la remoción de semillas de $B$. alicastrum por cada una de las dos condiciones de manejo se colocaron 12 unidades experimentales de $1.5 \times 1 \mathrm{~m}$ (72 encierros en total) en tres tratamientos. El primer tratamiento consistió en rodear la parcela con malla para gallinero $(1 \mathrm{~cm}$ de luz y $90 \mathrm{~cm}$ de altura), dejando en la base de la malla seis aperturas de 10 $\times 10 \mathrm{~cm}$. Este tratamiento sirvió para evaluar el efecto de los roedores pequeños aislando a los mamíferos medianos 


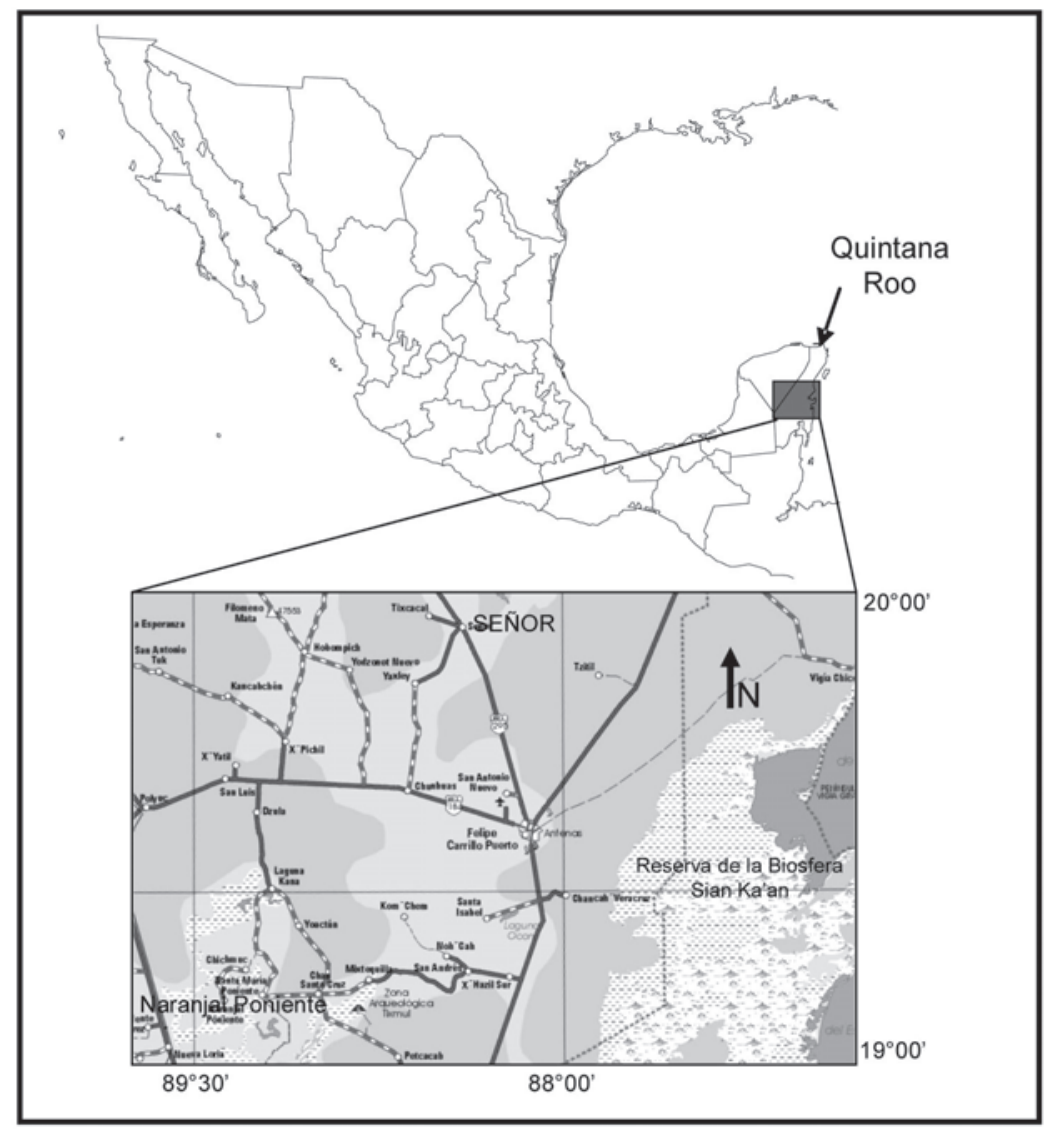

Figura 1. Ubicación de los dos ejidos forestales donde se realizó el estudio dentro de la Zona Maya de Quintana Roo, México

y grandes (MMG). El segundo tratamiento fue un testigo abierto el cual permitió el acceso tanto a mamíferos pequeños como grandes (TA). El tercer tratamiento fue un testigo cerrado (TC) el cual permitió tener un testigo contra el cual contrastar la remoción bajo el supuesto de una total exclusión de mamíferos terrestres. Se colocaron 20 semillas por tipo de encierro (1440 semillas en total). Las semillas fueron colectadas directamente debajo de la copa de 5 árboles localizados en una de las zonas de estudio (Señor). Para determinar la viabilidad de las semillas éstas fueron puestas a flotar en agua. Con este método se descartaron todas aquellas semillas que flotaran, ya que se considera que al no desarrollar por completo el embrión la densidad específica de estas es menor que aquellas que son viables, y por lo tanto flotan.

De las 20 semillas colocadas inicialmente todas aquellas semillas que no fueron removidas o depredadas in situ fueron seguidas hasta su germinación y posteriormente marcadas como plántulas. Aproximadamente un mes después se cuantificó la herbivoría en las plántulas supervivientes y un año después los individuos supervivientes fueron re-localizados y con estos se estimó el reclutamiento de B. alicastrum hasta esta categoría de edad. Para estimar la herbivoría se cuan- tificó el daño en todas las hojas presentes en los individuos reclutados (1154 hojas en total). Para el análisis se utilizó un índice de herbivoría puntual (IH) basado en seis categorías de daño en la lámina foliar (Dirzo y Domínguez, 1995): 0 = intacta; $1=<6 \% ; 2=6-12 \% ; 3=12-25 \% ; 4=25-50 \%$ y $5=>50 \%$. Así el IH se define como:

$\mathbf{I H}=\Sigma\left(\mathbf{n}_{\mathbf{i}} / \mathbf{i}\right) / \mathbf{N}$, donde:

ni= número de hojas en la categoría $\mathrm{i}$

$\mathrm{ci}=$ categoria de daño

$\mathrm{N}=$ número total de hojas

Análisis estadísticos. Con el fin de conocer si existieron diferencias en la remoción de semillas de $B$. alicastrum a través del tiempo, se utilizó el modelo de supervivencia log-rank, el cual es un método no paramétrico que permite hacer comparaciones entre diferentes curvas de supervivencia (Clark et al., 2003). De esta manera se contrastó la supervivencia en cada condición de manejo y la respuesta a los tratamientos experimentales. En el caso de las comparaciones entre sitios de manejo y exclusiones experimentales los datos no cumplieron con los supuestos estadísticos para utilizar estadística paramétrica (normalidad y homocedasticidad), por lo tanto se utilizaron modelos lineales generalizados, donde 


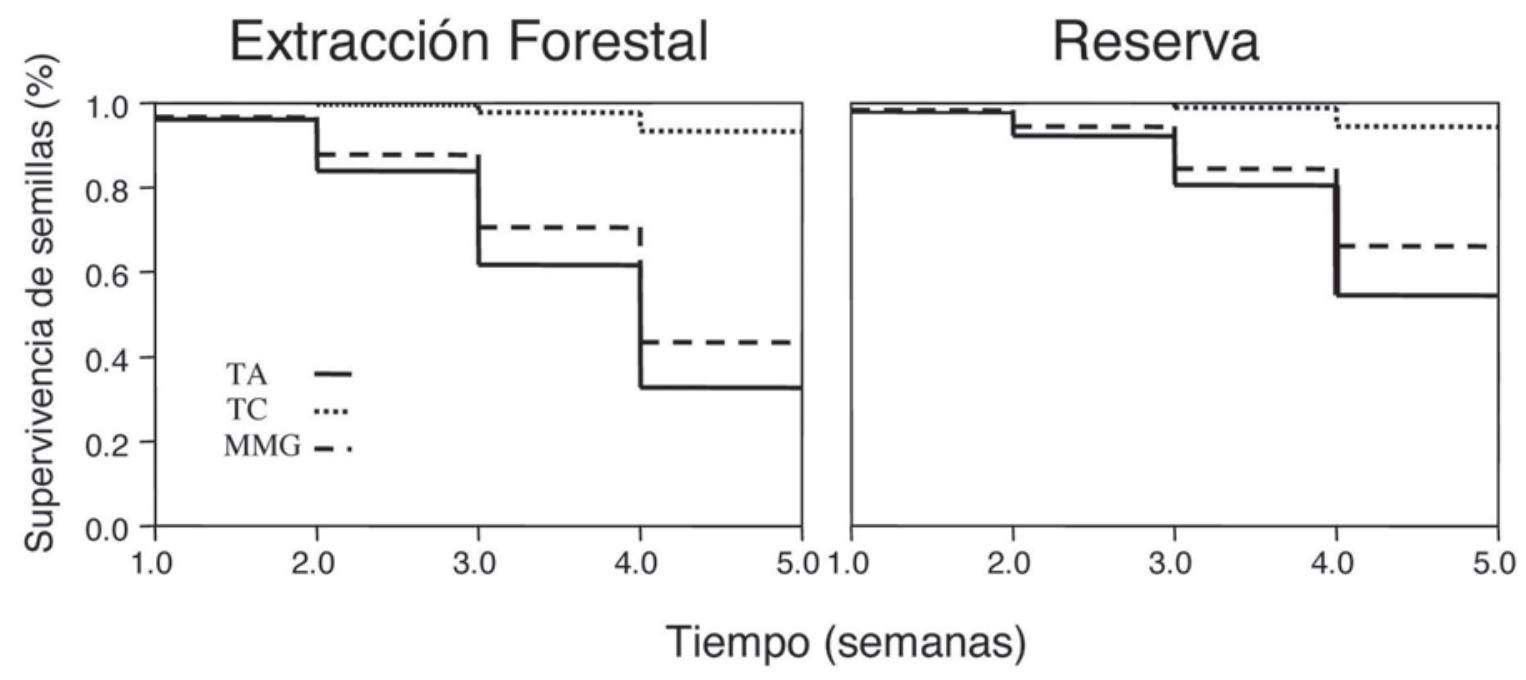

Figura 2. Supervivencia de semillas de Brosimum alicastrum en las dos condiciones de manejo y exclusiones experimentales. TA $=$ Testigo abierto; $\mathrm{TC}=$ Testigo cerrado; $\mathrm{MMG}=$ Exclusión de mamíferos medianos y grandes.

la variable de respuesta fue el número de individuos reclutados y los factores fueron: tipo de manejo (extracción forestal; reserva) y tratamiento experimental (MMG; TA; TC). En el caso del contraste entre tipo de manejo y la intensidad de la herbivoría los análisis fueron hechos agrupando todos los individuos reclutados por cada tipo de manejo ya que el número de individuos reclutados fue bajo. Se realizó un análisis de co-varianza, con el fin de determinar si la relación entre la abundancia de individuos y la herbivoría estaba en función de la condición de manejo. Posteriormente, para evaluar la existencia de denso-dependencia en la incidencia de herbivoría se utilizó un análisis de regresión entre la herbivoría y el número total de individuos reclutados en cada parcela experimental por condición de manejo. Todos los análisis fueron considerados estadísticamente significativos con una $P<0.05$. Los análisis fueron llevados a cabo con STATISTICA 7.0 (Stat Soft Inc., 2004).

\section{Resultados}

Remoción de semillas. Del total de semillas colocadas por tipo de manejo (720) un $34 \%$ no fueron removidas en las zonas de extracción forestal y un $56 \%$ en las zonas de reserva, la remoción de semillas fue más rápida en los sitos de extracción forestal (log-rank $\chi^{2}=915.5$, g.1. $2, P<0.0001$; Fig. 2). En cuanto a los tratamientos de exclusión las diferencias fueron estadísticamente significativas en los tratamientos con acceso de vertebrados $(P<0001)$. En el testigo abierto (TA) se registró la menor permanencia de semillas en ambas condiciones de manejo, en las zonas de extracción forestal fue en promedio de $0.9 \pm 1.3$ y en las de reserva de $5.5 \pm 3.3 \%$. En el caso del tratamiento donde se excluyen los mamíferos medianos y grandes (MMG) la permanen- cia de semillas fue en promedio de $10.6 \pm 2.4$ días en la zona de extracción forestal y de $12.25 \pm 3.2$ días en las de reserva. El testigo cerrado (TC) no presentó daños evidentes por roedores, pero un $12 \%$ de las semillas colocadas fueron depredadas in situ por insectos, no existieron diferencias estadísticas entre condiciones de manejo ( $P>0.05$; Fig. 3$)$.

Reclutamiento. Un total de 50 plántulas en las zonas de extracción forestal y 58 plántulas en las de reserva emergieron de las semillas que permanecieron sin remover, no existieron diferencias significativas entre ambas condiciones de manejo $(P>0.05)$. Sin embargo, sí existieron diferencias en el es-

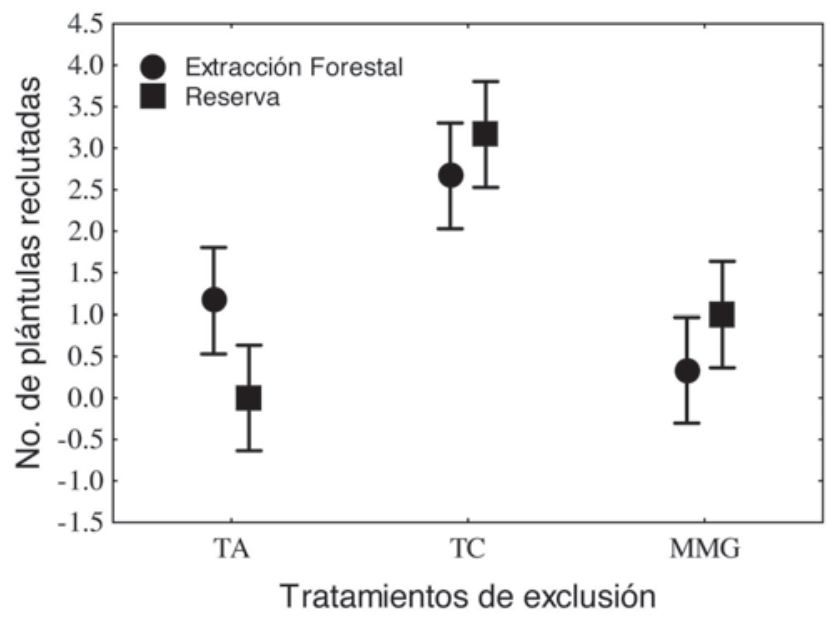

Figura 3. Plántulas reclutadas ( \pm D.E.) de Brosimum alicastrum en las dos condiciones de manejo y exclusiones experimentales. TA = Testigo abierto $;$ TC $=$ Testigo cerrado $;$ MMG = Exclusión de mamíferos medianos y grandes. 


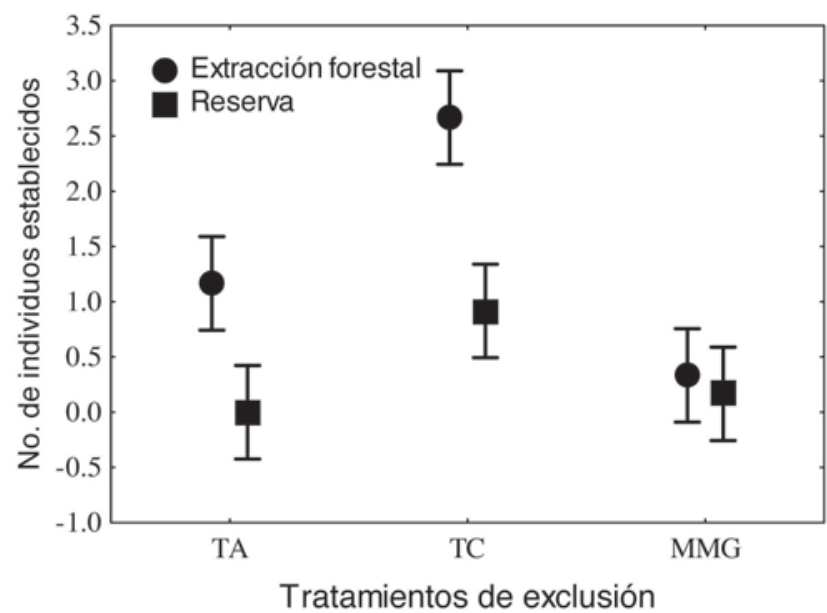

Figura 4. Individuos establecidos ( \pm D.E.) de Brosimum alicastrum en las dos condiciones de manejo y exclusiones experimentales. $\mathrm{TA}=$ Testigo abierto; $\mathrm{TC}=$ Testigo cerrado $\mathrm{MMG}=$ Exclusión de mamíferos medianos y grandes.

tablecimiento de plántulas entre tratamientos de exclusión. El mayor número de reclutamientos (38) se dio en el tratamiento que protegía a las semillas del consumo por vertebrados $(F=34.4$, g.1. 2,66, $P<0.0001$; Fig. 3). La interacción entre condición de manejo y tratamientos de exclusión fue significativa $(F=5.1$, g.1. $2,66, P<0.001)$, esto sugiere que las diferencias entre tratamientos depende de la condición de manejo.

Un año después del establecimiento de las plántulas de B. alicastrum, en los individuos ya reclutadas se presentaron diferencias significativas en el número de individuos sobrevivientes. Un total de 50 individuos sobrevivieron en las zonas de extracción forestal, mientras que 13 individuos lo hicieron en las zonas de reserva $(F=35.2$, g.1. 2,66, $P$ $<0.0001)$. La supervivencia de las plántulas fue mayor en aquellas plántulas protegidas de los vertebrados $(F=29.2$, g.1. 2,66, $P<0.001$; Fig. 4), y la interacción entre estos dos factores fue significativa $(F=7.6$, g.1. 2,66, $P<0.001)$, lo que confirma el patrón encontrado en el primer censo.

Herbivoría. El porcentaje de herbivoría en las zonas de extracción forestal fue del $4.6 \%$ y en las zonas de reserva del $6.2 \%$. Existió un mayor número de hojas sin daño aparente en las zonas de extracción forestal (175) que en las zonas de reserva (74), pero en general en ambas condiciones el daño foliar se concentró en las tres primeras categorías de daño (<25\% de daño). El resultado del análisis de covarianza mostró que existe una relación positiva y significativa donde un $42 \%$ de la variación en el daño foliar está explicado por la abundancia de individuos de B. alicastrum $(F=7.03$, g.1. 2,19, $P<0.05)$. Esta variación está significativamente relacionada con el tipo de manejo $(F=13.08$, g.1. $1,19, P$ $<0.05)$. Al analizar de manera separada por categoría de manejo la relación densodependiente entre la abundancia de los individuos reclutados y la herbivoría, se documentó que en las zonas de reserva existe una relación positiva y significativa entre las dos variables $\left(\mathrm{r}^{2}=0.79, \mathrm{n}=12, P<\right.$ 0.001 ) y con una pendiente $>1$, lo que sugiere la existencia de denso-dependencia. Por el contrario en las zonas de extracción forestal la relación no fue significativa $\left(\mathrm{r}^{2}=0.23, \mathrm{n}\right.$ $=12, P>0.05)$ y la pendiente fue $<1$, lo que indica ausencia de denso-dependencia (Fig. 5). Entre ambas pendientes existieron diferencias significativas $(F=13.3$ g.1. 1,20, $P<$ $0.001)$.

\section{Discusión}

Nuestros resultados muestran que a pesar de que existió una mayor remoción de semillas de Brosimum alicastrum en las zonas de extracción forestal, fue en estos sitios donde se dió un mayor reclutamiento de esta especie y que estos resultados están asociados a un cambio en los efectos densodependientes que puede presentar la herbivoría. Estos datos sugieren que a pesar de que la depredación de semillas relativamente pequeñas $(<1 \mathrm{~cm})$ es mayor, factores posteriores a este filtro también son modificados por las actividades forestales promoviendo un mayor establecimiento de B. alicastrum, en la zona de extracción forestal, tal y como sucede con otras especies como es Manilkara zapota (Gutiérrez-Granados y Dirzo, datos no publicados).

En el caso de la remoción de semillas, experimentos en los mismos sitios, pero con otras especies de semillas (Gutiérrez-Granados, datos no publicados) sugieren la tendencia a que las semillas con tamaño $<1 \mathrm{~cm}$ sean altamente consumidas en las zonas de extracción forestal, mientras que las semillas mayores presentan una alta permanencia en estos sitios. Estos cambios están asociados a un aumento en las abundancias de los roedores pequeños y una disminución en la abundancia de los mamíferos medianos y grandes

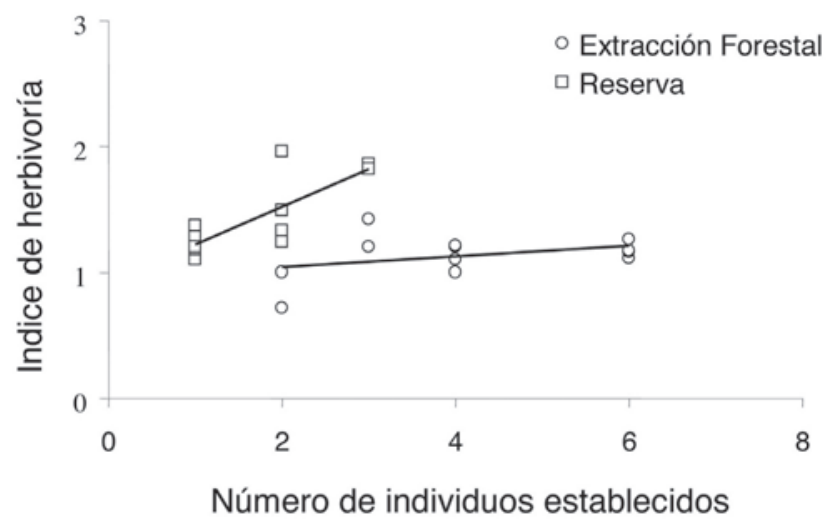

Figura 5. Relación entre el número de individuos establecidos de Brosimum alicastrum y el índice de herbivoría en las dos condiciones de manejo analizadas. 
(Gutiérrez-Granados, datos no publicados). Estos cambios en el escape de las semillas y juveniles de sus depredadores puede directamente la regeneración de la selva, como se ha reportado en otras zonas de los neotrópicos (DeMatia et al., 2006; Forget y Milleron, 1991; Sánchez-Cordero y Martínez-Gallardo, 1998; Stoner et al., 2007).

Si bien la variación en la remoción de semillas puede deberse a diversos factores inherentes a cada una de las especies, como pueden ser la calidad nutricional de las semillas y la presencia de defensas en éstas, abundancia o tipo de hábitat a donde son dispersadas para posteriormente ser removidas (Briones-Salas et al., 2006), algunos factores de origen humano también tienen influencia en la dinámica de la remoción de semillas. Actividades directas como la cacería o indirectas como la fragmentación del hábitat tienen influencia directa en las interacciones que establecen los mamíferos y las plantas (Writght, 2003). Sin embargo, la respuesta puede variar. Por ejemplo, Lambert y sus colaboradores (2005) registraron, en Brasil, que en algunas zonas con extracción de caoba, la remoción de semillas de caoba y cacahuates frescos no presentaba diferencias con zonas donde no se da este manejo. Asimismo, Guariguata y colaboradores (2000) documentaron que en zonas con alta fragmentación y extracción de madera, las semillas de Carapa nicaraguensis y Lecythis ampla, que son principalmente removidas por roedores grandes $(>1 \mathrm{~kg})$ permanecieron intactas. Estos datos sugieren que la remoción de semillas es un proceso altamente variable y que dependerá de la especie a remover (tamaño e historia natural), del removedor, del sistema natural donde se realice el proceso y del grado de perturbación que éste presente. Asimismo, la pérdida de semillas puede verse incrementada debido a la depredación realizada por insectos la cual en las selvas tropicales es alta (Howe, 1990; Notman y Gorchov, 2001).

En cuanto al establecimiento de las plántulas, Terborgh y Wright (1994) documentaron una mayor supervivencia de juveniles en zonas libres del acceso de mamíferos herbívoros, tal y como sucede con las plántulas de Brosimun alicastrum en la Zona Maya. En las zonas de extracción forestal generalmente se da una disminución en la abundancia de mamíferos herbívoros (Heydon y Bulloh, 1997), fenómeno que también ha sido documentado para otros bosques tropicales y en la Zona Maya (Meijaar et al., 2008; Gutiérrez-Granados, datos sin publicar) lo que puede explicar, en parte, la mayor supervivencia de juveniles. Sin embargo, no explica la mortalidad en los tratamientos sin acceso de mamíferos en las zonas de reserva. Las plántulas supervivientes sufren la influencia de factores denso-dependientes, como son: el ataque por enemigos naturales, encuentro con mutualistas y la intensidad de la competencia (Uriarte et al., 2004). Cuando estos factores tienen influencia sobre los juveniles de las plantas juegan un papel importante en la coexistencia de especies y mantenimiento de la diversidad vegetal (Chesson, 2000; Tilman, 2004), por lo que cualquier actividad que afecte directamente la remoción de semillas y la herbivoría en las selvas tropicales, como muestran los resultados obtenidos en las zonas de extracción forestal, favorecerá el establecimiento de ciertas especies lo que a largo plazo puede verse reflejado en una pérdida en la diversidad de las selvas (DeMattia et al., 2006; Stoner et al., 2007b).

La herbivoría es una de las presiones de selección que más influyen en la dinámica de las plántulas de una selva tropical. Sin embargo, ha sido poco estudiada la respuesta que ésta tiene a las actividades humanas (Benitez-Malvido et al., 1999). En las zonas de extracción forestal la abundancia de insectos herbívoros aumenta (Fredericksen y Fredericksen, 2002), y se ha especulado que las plantas pueden ser más palatables (Mooney y Gulmon, 1982), lo que permitiría pensar que la herbivoría aumentaría. Sin embargo, en la Zona Maya se documentó que existe un menor daño foliar en las zonas de extracción forestal, en comparación con las de reserva, y sin ninguna relación aparente con la abundancia de plántulas de Brosimum alicastrum, mientras que en las zonas de reserva el análisis de regresión indica que sí se mantiene una denso-dependencia del proceso. Esto sugiere que la extracción forestal puede liberar a las plántulas de $B$. alicastrum de la denso-dependencia y favorecer con esto un aumento en la densidad general de esta especie, tal y como se ha demostrado con modelos para la palma Euterpe edulis (Freckleton et al., 2003). Si bien, los datos sugieren que la extracción forestal puede alterar los mecanismos denso-dependientes, esto requiere de análisis futuros para determinar la intensidad de este efecto.

Implicaciones para la conservación. Terborgh y Wright (1994), documentaron de manera experimental, que las especies de semillas que fueron protegidas de la depredación por vertebrados presentaron una mayor supervivencia. En las áreas de extracción forestal de la Zona Maya la abundancia de los mamíferos medianos y grandes se encuentra disminuida (Gutierrez-Granados; datos no publicados), bajo este escenario y con los datos generados en este trabajo se puede esperar que la ausencia de los mamíferos terrestres de esta talla y las actividades que ellos realizan (ramoneo y pisoteo) afecte de manera diferencial el reclutamiento de algunas especies (DeMattia et al. 2006). Sí aunado a esto, los individuos reclutados en altas densidades son liberados de los procesos denso-dependientes es factible predecir que existirá un aumento en la representación de algunas especies como es el caso de Brosimum alicastrum lo que a largo plazo puede desencadenar un cambio en la diversidad vegetal y estructura de las selvas bajo manejo forestal.

\section{Agradecimientos}

Este trabajo se llevo a cabo gracias al apoyo económico otorgado por la CONABIO a través del proyecto BJ005. Deseamos agradecer el apoyo en el trabajo de campo a Os- 
car Hernández. GGG agradece al CONACYT y a la DGEPUNAM las becas otorgadas durante sus estudios doctorales, asimismo agradece el apoyo logístico al Posgrado en Ciencias Biológicas de la UNAM.

\section{Literatura citada}

Alavalapati J.R.R. y Zarin D. J. 2004. Neotropical working forests. For what and for whom. En: Zarin D.J., Alavalapati J.R.R., Putz F. y Schmink M. Eds. Working forest in the Neotropics. Conservation through sustainable management?, pp. 279-289, Columbia University Press, New York.

Benitez-Malvido J., García-Guzman G. y Kossmann-Ferraz I.D. 1999. Leaf-fungal incidence and herbivory on tree seedlings in tropical rainforest fragments: an experimental study. Biological Conservation 91:143-150.

Bray D.B., Merino-Pérez L., Negreros-Castillo P., Segura-Warnholtz G., Torres-Rojo J.M. y Vester H.F.M. 2003. Mexico's community-managed forests as a global model for sustainable landscapes. Conservation Biology 17:672-677.

Briones-Salas M., Sánchez-Cordero V. y Sánchez-Rojas G. 2006. Multi-species fruit and seed removal in a tropical deciduous forest in Mexico. Canadian Journal of Botany 84:433-442.

Carlson W.P. y Root R.B. 2000. Herbivory and plant species coexistence: community regulation by an outbreaking phytophagous insect. Ecological Monographs 70:73-99.

Clark T.G., Bradburn M.J., Love S.B. y Altman D.J. 2003. Survival analysis part I: Basic concepts and first analyses. British Journal of Cancer 89:232-238.

Chesson P. 2000. Mechanisms of maintenance of species diversity. Annual Review of Ecology and Systematics 31:343-366.

DeMattia E.A., Rathcke B.J., Curran L.M., Aguilar R. y Vargas O. 2006. Effects of small rodent and large mammal exclusion on seedling recruitment in Costa Rica. Biotropica 38:196-202.

DeMattia E.A., Curran L.M. y Rathcke J. 2004. Effects of small rodents and large mammals on Neotropical seeds. Ecology 85:2161-2170.

Dirzo R. 1984. Herbivory, a phytocentric overview. En: Dirzo R. y Sarukhan J. Eds. Perspectives in plant population biology, pp. 279-289, Sinauer Assoc. Inc. Publ., Sunderland, Mass, USA.

Dirzo R. y Dominguez C.A. 1995. Plant-herbivore interactions in Mesoamerican tropical dry forests. En: Bullock S.H., Medina E. y Mooney H.A. Eds. Seasonally dry tropical forests, pp. 304325, Cambridge University Press, Cambridge.

Forget P-M. y Milleron T. 1991. Evidence of secondary seed dispersal by rodents in Panama. Oecologia 87:596-599.

Freckleton R.P., Silva-Matos D.M., Bovi M.L.A. y Watkinson A.R. 2003. Predicting the impacts of harvesting using structured population models: the importance of density-dependence and timing of harvest for a tropical palm tree. Journal of Applied Ecology 40:846-858.

Fredericksen N.J. y Fredericksen T.S. 2002. Terrestrial wildlife responses to logging and fire in a Bolivian tropical humid forest. Biodiversity and Conservation 11:27-38.

Guariguata M.R., Rosales J.J. y Finegan B. 2000. Seed removal and fate in two selectively-logged lowland forest with contrasting protection levels. Conservation Biology 14:1046-1054.

Hall J., Harris D.J., Medjibe V. y Asthon P.M. 2003. The effects of selective logging on forest structure and tree species com- position in a Central African forest: implications for management of conservation areas. Forest Ecology and Management 183:249-264.

Heydon M.J. y Bulloh P. 1997. Mousedeer densities in a tropical rainforest: the impact of selective logging. Journal of Applied Ecology 34:484-496.

Howe H. 1990. Seed dispersal by birds and mammals: Implications for seedling demography. En: Bawa K.S. y Handley M. Eds. Reproductive Ecology of tropical forest plants, pp. 191218, UNESCO and Parthenon Publishing Group, Paris.

Janzen D. 1970. Herbivores and the number of tree species in tropical forests. American Naturalist 104:501-528.

Johns J.S., Barreto P. y Uhl C. 1996. Logging damage during planned and unplanned logging operations in the eastern Amazon. Forest Ecology and Management 89:59-77.

Jordano P., García C., Godoy J.A. y García-Castaño J.L. 2007. Differential contribution of frugivores to complex seed dispersal patterns. Proceedings of the National Academy of Sciences 104: 3278-3282.

Lambert T.D., Malcolm J.R. y Zimmerman B.L. 2005. Effects of mahogany (Swietenia macrophylla) logging on small mammal communities, habitat structure, and seed predation in the southeastern Amazon Basin. Forest Ecology and Management 206:381-398.

Makana J-R. y Thomas S.C. 2005. Effects of light gaps and litter removal on the seedling performance of six African timber species. Biotropica 37:227-237.

Malcolm J.R. y Ray J.C. 2000. Influence of timber extraction routes on Central African small-mammal communities, forest structure, and tree diversity. Conservation Biology 14:16231638.

Marquis R.J. 1984. Leaf herbivores decrease fitness of a tropical plant. Science 226:537-539.

Meijaard E., Sheil D., Marshall A.J. y Nasi R. 2008. Phylogenetic age is positively correlated with sensitivity to timber harvest in bornean mammals. Biotropica 40:76-85.

Mendoza E. y Dirzo R. 2007. Seed-size variation determines interspecific differential predation by mammals in a neotropical rain forest. Oikos 116: 1841-1852.

Mooney H.A. y Gulmon S.L. 1982. Constrains on leaf structure and function in reference to herbivory. BioScience 32:198-206.

Muller-Landau H.C., Wright S.J., Calderon O., Hubbell S.P. y Foster R.B. 2001. Assessing recruitment limitation: Concepts, methods and case-studies from a tropical forest. En: Levey D.J., Silva W.R. y Galetti M. Eds. Seed dispersal and frugivory: Ecology, evolution and conservation, pp. 34-54, CABI Publishing, Wallingford.

Notman E. y Gorchov D.L. 2001. Variation in post-dispersal seed predation in mature Peruvian lowland tropical forest and fallow agricultural sites. Biotropica 33:621-636.

Ochoa G.J. 2000. Efectos de la extracción de maderas sobre la diversidad de mamíferos pequeños en bosques de tierras bajas de la Guayana venezolana. Biotropica 32:146-164.

Olmsted I. y Durán R. 1990. Vegetación de Sian Ka’an. En: Navarro D.L. y Robinson J.G. Eds. Diversidad Biológica en la reserva de la Biosfera de Sian Ka'an Quintana Roo, México, pp. 1-13, Centro de Investigaciones de Quintana Roo. México.

Peres C.A. 2001. Synergistic effects of subsistence hunting and habitat fragmentation on Amazonian forest vertebrates. Conservation Biology 15:1490-1505. 
Rodríguez-Velázquez J., Martínez-Rámos M. y Dyer G. 1997. Brosimum alicastrum (ojoche). En: Gonzalez-Soriano E., Dirzo R. y Vogt R.C. Eds. Historia Natural de Los Tuxtlas, pp. $97-$ 100, Instituto de Biología, Instituto de Ecología y CONABIO. México.

Sánchez-Cordero V. y Martínez-Gallardo R. 1998. Postdispersal fruit and seed removal by forest-dwelling rodents in a lowland rainforest in Mexico. Journal of Tropical Ecology 14:139-151.

Schmitz O.J. 2008. Herbivory from individuals to ecosystem. Annual Review in Ecology, Evolution and Systematics 39:133-152.

Stat Soft Inc. 2004. STATISTICA (Data analysis software system) versión 7. www.statsoft.com

Stoner K.E., Riba-Hernández P., Vulnec, K. y Lambert J.E. 2007. The role of mammals in creating and modifying seedshadows in tropical forests and some possible consequences of their elimination. Biotropica 39:316-327.

Stoner K.E., Vulnec K., Wright S.J. y Peres C.A. 2007b. Hunting and plant community dynamics in tropical forests: A synthesis and future directions. Biotropica 39:385-392.

Terborgh J.W. y Wright S.J. 1994. Effects of mammalian herbivores on plant recruitment in two Neotropical forests. Ecology 75:1829-1833.

Tilman D. 2004. Niche tradeoffs, neutrality, and community struc- ture: A stochastic theory of resource competition, invasion, and community assembly. Proceedings of the National Academic of Science 101: 10854-10861.

Uriarte M., Condit R., Canham C.D. y Hubbell, P.S. 2004. A spatially explicit model of sapling growth in a tropical forest: Does the identity of neighbors matter? Journal of Ecology 92:348360.

Webb E.L. 1997. Canopy removal and residual stand damage during controlled selective logging in lowland swamp forest of northeast Costa Rica. Forest Ecology and Management. 95:117-129.

Wright S.J. y Duber H.C. 2001. Poachers and forest fragmentation alter seed dispersal, seed survival and seedling recruitment in the palm Attalea butyraceae, with implications for tropical tree diversity. Biotropica. 33:583-595.

Wright S.J., Zeballos H., Domínguez I., Gallardo M., Moreno M.C. y Ibañez R. 2000. Poachers alter mammal abundance, seed dispersal and seed predation in a Neotropical forest. Conservation Biology 14:227-239.

Wright S.J. 2003. The myriad consequences of hunting for vertebrates and plants in tropical forests. Perspectives in Plan Ecology, Evolution and Systematics 6:73-86.

Recibido: 9 de diciembre de 2008

Aceptado: 1 de julio de 2009 